\title{
DOSSIER
}

\section{SYLVIE CHEVRIER}

Université d'Evry Val d'Essonne; IRG

\section{JEAN-PIERRE SEGAL}

CNRS - Dauphine Recherche Management

\section{Coordination des équipes multiculturelles au sein des multinationales}

\section{Des acteurs en quête de modes d'emploi}

Comment s'opère la coordination au sein des nouvelles structures transversales que multiplient les firmes multinationales? Avec quelle efficacité? Aux enjeux de pouvoir entre siège et filiales s'ajoutent de nouvelles diversités, celle des langues et des repères culturels. Au détriment, dans le cas étudié, de l'efficacité de ces structures de coordination mais aussi de l'intégration des nouvelles unités dans le Groupe. 
$\mathrm{L}$ e potentiel de synergies que recèlent les firmes multinationales ne peut se concrétiser qu'à la condition d'une coordination efficace entre leurs entités. Pour espérer bénéficier d'économies d'échelle et d'un pouvoir de marché important, pour pouvoir tirer partie de la diversité culturelle, pour se maintenir à l'avant-garde de l'innovation, siège et filiales doivent coordonner la mobilisation des compétences détenues par des experts désormais dispersés (Persson, 2006). Certes, les difficultés de coordination ne sont pas propres aux firmes multinationales. Dès les années 1960, Lawrence et Lorsch avaient mis en exergue la tension entre différenciation et intégration et souligné les enjeux et les difficultés de la coordination dans les organisations multidivisionnelles. Cependant, l'internationalisation des entreprises apporte une somme de difficultés supplémentaires. Aux classiques conflits de pouvoir et d'intérêts des entités autour de la défense de leur autonomie ou de l'obtention de ressources s'ajoutent désormais de nouveaux enjeux liés à la gestion des obstacles que constituent la distance physique, la pluralité des langues, les différences culturelles et l'hétérogénéité des organisations, en termes de taille, de structure et de moyens (Keup et Gassman, 2009). Cet article se propose donc d'examiner une question ancienne - la mise en œuvre de la coordination - dans un contexte nouveau, celui de la firme multinationale, arrangement organisationnel complexe qui résulte le plus souvent d'une série d'acquisitions, de fusions et de diverses formes d'associations avec des partenaires étrangers.

La littérature en gestion distingue différentes formes de coordinations, formelles et informelles, dans les firmes multinationales (Huault, 1998).
La coordination s'appuie d'abord sur des structures transversales. Ainsi, la tension entre centralisation et décentralisation étudiée le plus souvent à travers les relations siège-filiales se résout par la mise en place d'une structure en réseau qui favorise la circulation des connaissances et savoir-faire (Nohria et Ghoshal, 1997).

La coordination passe également par des dispositifs de gestion partagés; il s'agit de s'assurer que l'ensemble des entités utilise les mêmes outils et les mêmes standards à travers la diffusion de normes et de repères communs. La mise en place des progiciels de gestion intégrés qui structurent de manière homogène l'information occupe une place centrale dans ces stratégies nouvelles d'intégration (Clemmons et Simon, 2001). La standardisation attendue est censée faciliter échanges et communication. Le partage des valeurs que la maison-mère codifie généralement sous formes de charte éthique ou de principes d'action, constitue un troisième levier de coordination. Cette coordination informelle entend promouvoir le partage d'une vision et de valeurs communes censées orienter les décisions des salariés de toutes les entités vers la même direction. L'objet est de créer une « culture de groupe ", en s'appuyant sur des formations mais aussi en encourageant la circulation et la socialisation des salariés à travers des expatriations et impatriations (Schaaper, 2005). L'adoption de l'anglais comme langue véhiculaire commune accompagne généralement cette initiative. Ces méta-coordinations restent pourtant virtuelles tant que les acteurs ne les mettent pas en œuvre. La création d'une structure transversale (projet, comité de pilotage) ne garantit pas la qualité de son fonctionnement (Moennaert et al., 2000). De même, 
l'analyse concrète des transferts d'outils de gestion a montré les limites de la diffusion de normes communes et l'éventuelle hybridation voire divergence des pratiques entre filiales (Kostova et Roth, 2022). Quant à la diffusion des valeurs de l'entreprise à travers des chartes éthiques, elle suscite réactions mitigées et réinterprétations majeures (Barmeyer et Davoine, 2004; d'Iribarne, 2009). Même l'anglais imposé comme langue commune suscite des réticences (Piekkari et al., 2005). La qualité de la coordination dans les firmes multinationales se joue donc sur le terrain dans l'interaction entre les acteurs et dans leur manière de s'approprier les démarches d'intégration impulsées. L'efficacité de la coordination apparaît comme une question non seulement technique mais aussi sociale et politique compte tenu des processus de désorganisation et de résistance (Morgan, 2001).

Or, les modes informels de coordination et les microrégulations interpersonnelles sont généralement analysés sous le seul angle des difficultés de communication interculturelle entre siège et filiales (Clausen, 2010), sans prêter suffisamment attention aux contraintes organisationnelles et politiques qui pèsent sur ces échanges. Cet article propose d'examiner comment les structures transversales au sein d'entreprises multinationales tentent de s'arranger de telles contraintes, prenant le parti de soumettre à l'épreuve des faits les bénéfices espérés de leur diversité renforcée. Sans négliger les difficultés communes à toutes les structures transversales (conflits d'intérêt, de pouvoir, pénurie de ressources disponibles, ambiguité des mandats, déficit de leadership, implication incertaine des participants), nous analysons avec une attention toute particulière comment ces structures composent, au sein des firmes multinationales, avec les interfaces culturelles, autrement dit avec la diversité des représentations qui les traversent.

\section{MÉTHODOLOGIE DE LA RECHERCHE}

Cet article s'appuie sur les résultats d'une recherche ethnographique analysant, au sein d'un Groupe international basé en France, le fonctionnement de trois structures transversales de coordination réunissant des représentants du siège et des principales filiales étrangères. Ces structures, relevant de chacune des trois grandes divisions de l'entreprise, fonctionnent au moment de l'étude depuis au moins six mois et font travailler ensemble au moins trois nationalités différentes.

Les matériaux réunis comprennent des entretiens, en face à face ou par téléphone, en français ou en anglais, avec une quarantaine d'acteurs de différentes nationalités auxquels s'ajoutent plusieurs observations de réunions de travail, physiques et à distance. Sans négliger l'impact des difficultés de communication liées aux maîtrises très inégales de l'anglais - langue de travail utilisée - l'analyse s'est surtout intéressée à la variété des interprétations que donnent les différentes nationalités en présence des difficultés rencontrées ainsi qu'à l'incidence de ces lectures contrastées sur le fonctionnement des structures transversales étudiées. Le tableau 1 ci-après présente un échantillon de l'enquête. 
Tableau 1 - Échantillon d'enquête

\begin{tabular}{|c|c|c|c|c|}
\hline & Cas 1 & Cas 2 & Cas 3 & Total \\
\hline Français & 5 & 6 & 8 & 19 \\
\hline Non-français & 6 & 11 & 6 & 23 \\
\hline Nationalités & $\begin{array}{c}\text { Britanniques } \\
\text { Polonais }\end{array}$ & $\begin{array}{c}\text { Belges } \\
\text { Espagnols }\end{array}$ & $\begin{array}{c}\text { Allemands } \\
\text { États-uniens }\end{array}$ & \\
\hline Structure centrale & 2 & 7 & 5 & 14 \\
\hline Antennes locales & 9 & 10 & 9 & 28 \\
\hline Total & 11 & 17 & 14 & 42 \\
\hline
\end{tabular}

\section{I - TROIS EXEMPLES DE \\ COORDINATION TRANSVERSALE}

Les thèmes sur lesquels les trois structures transversales étudiées travaillent illustrent l'étendue des sujets que ces groupes transversaux de coordination peuvent être amenés à traiter: intégration des systèmes d'information, fertilisation croisée dans la conception des offres commerciales et pilotage de la mise en œuvre dans plusieurs pays d'un contrat de services global.

\section{Cas 1 : construire un système d'information intégré}

Le premier des groupes de travail transversaux s'inscrit dans un projet d'intégration des systèmes d'information au sein d'une des branches de notre entreprise. Plusieurs groupes de convergence, chacun en charge d'un sujet spécifique, préparent les décisions qui seront prises à l'échelle du Groupe. La recherche a accompagné l'un d'entre eux. Leur mission est double: bâtir, d'abord, un cahier des charges synthétisant les attentes et les contraintes de l'ensemble des utilisateurs futurs du système d'information; puis, une fois l'architecture finale du système arrêté par la direction de branche, piloter le travail de construction de ces nouveaux outils intégrés.

Chaque filiale est donc censée participer à ces structures de convergence, soit en envoyant un représentant à Paris (ce que font généralement les Français) soit en suivant à distance les réunions qui se tiennent en anglais. Chaque représentant peut, en principe, y exprimer la spécificité de ses besoins, y présenter ses outils et proposer son expertise afin de fertiliser la réflexion transversale au Groupe. En pratique, les choses se passent assez différemment. " Beaucoup d'observateurs et peu de contributeurs », regrette l'animateur français du groupe. La confrontation entre la liste officielle des participants et les présents fait apparaitre un niveau élevé d'absentéisme. La partie britannique du Groupe est ici la plus impliquée, souhaitant obtenir des moyens supplémentaires pour développer ses propres projets et les proposer au reste du Groupe. Elle a pour ce faire délégué un consultant spécialement chargé de faire le lobbying de son projet. La partie française, qui voyait au départ dans ces structures de concertation l'ébauche de la construction d'une culture de groupe, s'étonne en privé du 
choix d'un ambassadeur « mercenaire ». Ce consultant, prêt à s'investir dans le fonctionnement du groupe et à seconder l'animateur français dans ses tâches de coordination, n'en est pas moins le bienvenu. Les Polonais, en mal de trouver des représentants à la fois anglophones et spécialistes des sujets débattus, sont marginalisés, réduits au statut d'observateur. La partie française, la plus largement représentée, serait parfaitement à l'aise avec ce dispositif si ses représentants maîtrisaient mieux la langue de travail. Restés silencieux pendant la réunion, ces derniers s'animent quand l'anglais, utilisé aussi longtemps que les correspondants étrangers sont en ligne, cède la place à la langue de Molière. La méthode de travail adoptée, qui consiste à organiser, dans un premier temps, des débats internes, vifs et argumentés si possible, permettant de faire émerger une véritable intelligence collective des problèmes « de fond » et, dans un second temps, à confier à la hiérarchie, éclairée par ces débats, le soin de faire les arbitrages qui relèvent de ses responsabilités, fait sens aux yeux des Français (d'Iribarne, 1998), beaucoup moins aux yeux des Britanniques qui expriment deux critiques principales: les conduites de réunion manquent à leurs yeux de professionnalisme, traînent en longueur et ne débouchent sur aucune décision concrète. Le système de décision « à la française ", surtout, leur paraît manquer singulièrement de transparence. «En tant que manager, nous dit le responsable de l'unité anglaise au cours de l'entretien bref et dense qu'il nous accorde, j'estime avoir mon mot à dire sur les choix qui impactent les résultats de ma business unit puisque je rends personnellement des comptes à ce sujet ». La concertation, telle que les Fran- çais l'entendent, est assimilée à un processus hypocrite d'enregistrement de décisions déjà prises. Le choix par le Groupe, converti à l'anglais, du terme de « Business Owner » pour désigner les responsables de filiales ou de services, se révèle trompeur aux yeux de ces derniers, dans la mesure où ils s'estiment privés des capacités de décision attachées au statut de «propriétaire » tel qu'ils le conçoivent.

Les participants français au groupe de convergence ne sont pas non plus satisfaits mais pour d'autres raisons. L'élargissement géographique, linguistique et culturel du périmètre d'action du Groupe les conduit à s'interroger sur la fécondité de la méthode utilisée au regard de la minceur des débats qui s'établissent. Ils font aussi leur deuil de la perspective de faire œuvre pionnière dans la construction, à la base, d'une vision partagée des problèmes entre les diverses composantes nationales du Groupe. Pendant ce temps la responsable de la composante polonaise associée au projet, nous déclare, dans son bureau de Varsovie où nous l'interrogeons, qu'elle profite de ces réunions, à ses yeux sans intérêt, pour " ranger ses armoires ». Loin d'avoir rapproché les points de vue, le fonctionnement de cette structure paraît avoir surtout nourri les frustrations des participants et alimenté leur méfiance réciproque: les Français soupçonnent les Britanniques de n'avoir toujours pas digéré leur absorption par le Groupe; ces derniers mettent en doute les compétences managériales des Français.

\section{Cas 2 : développer la fertilisation croisée des offres commerciales}

La seconde structure étudiée est une équipe de marketing stratégique chargée d'organi- 
ser la convergence des offres de services commercialisés auprès des clients de différentes filiales européennes. Le travail de cette équipe, jeune et polyglotte, consiste à identifier les besoins des clients finaux à l'échelle du Groupe et à favoriser les synergies potentielles entre filiales. Nous nous sommes attachés à suivre des projets faisant travailler ensemble, sous la houlette de la structure de coordination à composition essentiellement française, des équipes situées en France, en Belgique et en Espagne. Chacune de ces unités est mise à rude épreuve par les réajustements continuels des réunions, des déplacements et des points téléphoniques, qui sont sans cesse reportés ou avancés, du fait des multiples contraintes des participants. La construction de synergies entre des filiales s'efforçant chacune de coller aux attentes locales de ses marchés se révèle lourde de tensions. Les nombreuses itérations dans la négociation des caractéristiques des services à livrer aboutissent à des changements au cours même du processus de développement. Tandis qu'au siège la cellule de coordination se mobilise du mieux qu'elle peut pour tenir ses objectifs, beaucoup, dans les filiales, se plaignent de l'inefficacité de la coordination globale: " on a dû refaire en 15 jours, ce qu'on avait fait en 3 mois », déclare un membre de l'équipe espagnole. Ce n'est pourtant pas l'expérience internationale qui manque aux acteurs en cause, ni la maîtrise des langues étrangères dans laquelle la plupart d'entre eux excellent. Ce sont leurs conceptions d'une construction efficace de la coopération qui divergent.

La difficulté principale évoquée par les acteurs porte sur la mise en œuvre des contrats entre clients et fournisseurs internes.
Ainsi, du côté belge, l'un des points critiques renvoie à la difficulté d'obtenir la satisfaction de ses attentes en tant que client interne. Pour ces partenaires, la relation client-fournisseur est censée respecter une logique de marché: le client établit son cahier des charges, fait jouer la concurrence et, finalement, choisit les produits qui lui conviennent le mieux. En principe, la filiale locale pourrait recourir à un fournisseur externe; dans les faits, des pressions politiques venues du siège la contraignent à signer un contrat interne. Libres de leurs choix quand ils font affaire avec des prestataires externes, les partenaires belges, dès lors que leur demande est réexaminée au niveau du siège, se sentent privés de leurs prérogatives « normales » dans la négociation portant sur les prix, mais surtout sur la définition même du service. L'adoption de l'anglais comme langue de communication conduit la partie belge à utiliser le terme de « requirements » qui, appliqué à un client, exprime dans la langue de Shakespeare, l'impérieuse nécessité de respecter ses desiderata. L'équipe centrale de coordination voit les choses bien autrement. À ses yeux, la satisfaction des « demandes " locales ne peut se concevoir indépendamment du respect de l'intérêt général du Groupe, vu comme incontournable principe supérieur commun. Un tri est effectué par l'unité centrale entre les requêtes qui convergent et celles qui ne correspondent qu'à un marché spécifique. Ces dernières risquent fort de ne pas être retenues. Plus la demande va dans le sens des besoins des filiales qui ont du poids dans le Groupe, plus grandes sont ses chances d'être acceptées. Les « petites » filiales s'estiment donc injustement traitées et tentent de faire alliance avec d'autres petits pays. 
Même quand elle obtient du Groupe un accord sur le service qu'elle entend développer, la filiale belge ne se satisfait pas des manières d'agir des prestataires français du Groupe. Celles-ci heurtent ses conceptions de la « bonne manière » de traiter un client, fût-il interne. Les entités françaises s'autorisent à « reformuler » la demande et à la satisfaire de manière sélective. Par exemple, un service fondamental ayant été livré, les services périphériques attendus ont été jugés très secondaires par les Français sollicités au regard des "vraies » urgences. Cet arbitrage est interprété dans la filiale comme une marque de suffisance hexagonale et de non-respect des prérogatives du client, telles que les Belges les conçoivent. Un enquêté belge qui attendait ainsi d'une entité française documentation et formation explique avoir dû batailler pendant plus d'un mois pour finalement n'en obtenir qu'une partie: « J'ai dû expliquer de nombreuses fois mais ils ne comprenaient pas pourquoi on voulait ça. Ils nous disaient "à quoi ça va vous servir?" (...) Finalement, la documentation, on a dû la faire nous-mêmes et ils ont envoyé quelqu'un pour un training de base ».

Le contraste est également saisissant entre la souplesse des processus d'ajustements mutuels dans la filiale espagnole et la lourdeur des formes de coordination auxquelles l'équipe centrale, elle-même insérée dans une méta-structure, doit recourir. En Espagne, l'organisation en bureau paysager permet une libre circulation des personnes entre les bureaux, une facilité abondamment utilisée. Chacun n'hésite pas à se déplacer pour aller voir sans préalable particulier la personne concernée pour avancer directement avec elle sur le sujet et des réunions improvisées peuvent se tenir à tout moment. Au siège français, chacun travaille dans son bureau porte fermée. Les réunions doivent être programmées et aucune décision d'importance ne pourra faire l'économie d'un comité formel d'expertise. Les personnels des filiales n'ont que faire du fait que la structure centrale soit dans l'obligation d'aligner les actions qu'elle coordonne par rapport aux autres projets du Groupe. Vus d'Espagne, les délais requis pour obtenir une réponse des autres entités paraissent démesurés.

\section{Cas 3 : piloter le déploiement local d'un contrat international}

La troisième situation étudiée correspond au pilotage de l'exécution d'un contrat de services pour le compte d'un autre Groupe international français. Ce dernier a mis fin aux contrats qui le liaient, dans six pays, à des fournisseurs locaux pour privilégier un contrat global avec notre entreprise. La mise en œuvre de cet ambitieux contrat exige la coordination entre le groupe central de pilotage, qui assure en sus la liaison avec le donneur d'ordre à Paris, et des équipes opérationnelles qui, dans chacun des pays concernés, sont les interlocuteurs directs des filiales locales du client. Cellesci s'étaient adressées jusqu'ici à des fournisseurs concurrents. Elles ont été mises devant le fait accompli par leur tutelle française qui leur a imposé comme nouveau prestataire le Groupe que nous étudions. Ce dernier a dû parfois sous-traiter à l'ancien fournisseur la production locale tout en se portant garant vis-à-vis du client de la qualité de la prestation fournie. Or, la qualité du service rendu n'est pas toujours exemplaire, en raison notamment de la complexité du montage du contrat. Le contexte 
de mise en œuvre du contrat est donc particulièrement délicat. On s'est intéressé aux coordinations mises en place entre la structure centrale et les représentants de deux filiales importantes allemande et américaine, coordinations qui prennent la forme de conférences téléphoniques régulières.

Comme dans nos deux autres cas, les personnels des filiales étrangères s'interrogent sur la valeur ajoutée de la structure française de coordination et sur sa façon d'opérer. Les filiales locales sont placées en première ligne pour recevoir les griefs de leurs clients à l'égard du Groupe français, griefs à l'égard des « Français » qu'elles peuvent parfois partager. Les personnels jugent de concert avec leurs clients que les réclamations ne sont pas suffisamment prises en considération. La propension des responsables français à sacrifier sur l'autel des intérêts du Groupe certaines demandes particulières suscitent les mêmes irritations locales que dans notre cas précédent. Les représentants des filiales étrangères attribuent volontiers cette attitude à l'ancienne culture de monopole du Groupe français. Les clients allemands, par exemple, acceptent très mal les manquements aux engagements contractuels pris. Le partenariat client-fournisseur, tel que les Allemands le conçoivent, n'admet pas de telles défaillances. Le fournisseur n'a pas non plus à s'autoriser de sa compétence pour décider à la place du client comme le reconnaît un responsable français: « En France, on se déclare vite "embêté" par le client ou alors on lui annonce: “c'est nous qui sommes en charge; on va vous expliquer comment cela va se passer" "».

Pour sa part, l'unité centrale estime, en tant que responsable de la prestation globale, devoir disposer d'une « vision d'ensemble » du déroulement des opérations. À ce titre, elle compte sur ses antennes locales pour contrôler de près la qualité du service rendu mais elle ne limite pas sa mission à cette fonction classique de supervision. Elle ambitionne d'aller plus loin, en mutualisant les diverses expériences locales, en homogénéisant le traitement des incidents clients et en faisant partager entre les filiales les bonnes pratiques développées par chacune d'elles. Dans cette perspective, la structure centrale associe deux méthodes: elle adresse des tableaux de bords détaillés à chaque antenne pays, et elle organise, en parallèle, des conférences hebdomadaires dans le cadre desquelles la parole, en principe, est libre.

Le suivi des conférences téléphoniques hebdomadaires entre l'unité de pilotage et ses correspondants locaux révèle, comme dans le cas $n^{\circ} 1$, la concision des réponses locales aux sollicitations venues du siège et la difficulté à instaurer la qualité d'échanges escomptée par l'unité centrale. Le terme de " Business Community », que la structure centrale utilise, met l'accent sur l'exigence de partage entre les filiales et pourrait bien être porteur des mêmes ambigüités que le terme de Business Owner. Aux oreilles des Français, il évoque la communauté de métiers, partageant ses savoirs: " On est en train de créer des communautés, déclare le responsable français des opérations, permettant de se réunir autour d'un même type de problème plutôt que de se voir par pays ». Les filiales anglo-saxonnes entendent plutôt « communauté d'affaire », à l'écoute des « requirements » des clients locaux, que « communauté professionnelle ». La ressemblance, ici encore, est grande avec les préoccupations exprimées dans nos deux autres cas. On reproche aux Français de se consacrer davantage à l'analyse approfondie des problèmes plutôt qu'à leur traitement rapide et efficace. 
Les procédures de reporting, élaborées par la cellule centrale pour suivre précisément les opérations sont mal reçues par les personnels de la filiale américaine aux effectifs étroitement calculés. " Si nous rendons déjà compte à notre patron américain, s'étonne un enquêté américain, pourquoi rendre des comptes en plus aux Français? ». Ce sont finalement les formes de coordination, formelles et informelles, élaborées par la structure centrale qui sont contestées, jugées inutiles et chronophages, ébranlant, par là même, la légitimité de ceux qui les ont proposées.

\section{II - EXCEPTION OU CAS D'ÉCOLE?}

Notre firme multinationale, longtemps en situation de monopole public, traverse incontestablement une période troublée, devant gérer d'importantes restructurations dans son activité traditionnelle et, simultanément, développer de nouveaux services et de nouveaux produits sur un marché désormais hyperconcurrentiel. La voici tiraillée entre la nécessité de décentraliser vers ses filiales étrangères la partie « culture bound » de ses activités grand public, pour mieux se rapprocher de ses clients, tout en renforçant le contrôle exercé par le siège dans un contexte où la situation financière de l'entreprise l'oblige à donner aux marchés les réassurances attendues. La mise en place des synergies entre le Groupe et ses nouvelles acquisitions étrangères s'effectue donc dans un contexte particulièrement mouvant, marqué par d'incessantes réorganisations. Celles-ci sont souvent perçues comme désordonnées par des personnels soumis, tant au centre qu'à la périphérie du groupe, à une forte pression. Pour avoir souhaité restituer nos résultats de recherche auprès de chacun des groupes de travail étu- diés, nous avons pu faire nous-mêmes l'expérience des difficultés et des frustrations engendrées par l'organisation de tels échanges internes. L'absentéisme constaté dans le cadre de ces réunions, les retards et les contretemps subis pour les mettre sur pied, les contributions très inégalement réparties entre Français et non-Français montraient bien combien les conditions requises pour établir des échanges féconds au sein du Groupe étaient difficiles à réunir. Contrairement à d'autres firmes internationalisées de longue date et disposant en leur sein des compétences linguistiques et culturelles requises pour faire face à ce type de situation, notre entreprise effectue sur le tas son apprentissage des exigences requises pour construire, à l'échelle élargie de son nouveau périmètre, des coopérations transnationales efficaces. Le fait que le Groupe ait un passé de monopole public et que l'État possède encore une minorité de contrôle de son capital, interfère, enfin, avec les représentations que s'en font les filiales étrangères, surtout quand elles appartiennent à un monde anglo-saxon éloigné des références françaises en matière de management (Barsoux et Lawrence, 1990). Certes, les difficultés mentionnées n'affectent pas uniquement les multinationales d'origine française. Une recherche conduite dans une banque sur les relations entre une filiale britannique et le siège situé en Allemagne met en évidence des récriminations de la part des salariés à Londres à propos de la vision « germano-centrée » du siège et de son fonctionnement bureaucratique et hiérarchique (Moore, 2005). Quel que soit le pays d'origine, les relations siège - filiales sont marquées par la géopolitique, l'histoire et les stéréotypes nationaux associés, toujours susceptibles de mettre en question la 
légitimité des pratiques managériales du siège. Cependant, plusieurs des constatations faites recoupent les enseignements de travaux comparatifs antérieurs (d'Iribarne et al., 1998) effectués au sein des filiales étrangères d'entreprises françaises. Qu'il s'agisse des modalités adoptées dans la prise de décision, dans la conception du rôle du manager ou dans la façon d'envisager les rapports avec les clients, s'observent chez les acteurs français de nos trois cas, une identité de vue remarquablement proche de celles observées par ailleurs dans d'autres multinationales françaises, au passé public ou privé (d'Iribarne, 2009).

\section{III - UN PRIX À PAYER ÉLEVÉ POUR FONCTIONNER « MALGRÉ TOUT »}

À défaut de susciter toutes les synergies espérées, les structures transversales étudiées parviennent néanmoins à fonctionner a minima: les projets voient le jour, les services sont fournis aux clients et les contrats sont mis en œuvre. Cependant, les résultats obtenus doivent davantage au surinvestissement de quelques-uns, en particulier ceux qui doivent personnellement rendre compte, qu'à l'intégration réussie de la contribution de tous. $\mathrm{La}$ métaphore, utilisée par un de nos répondants, du cygne qui glisse majestueusement sur l'eau tandis qu'à l'abri des regards s'agitent furieusement ses pattes, résume plaisamment la situation vécue par les acteurs.

En termes de climat, l'évitement et les tensions latentes sont bien plus fréquents que les conflits ouverts. Dans les trois cas, les acteurs internes préfèrent mettre les dysfonctionnements sur le compte de la distance, de la rareté des réunions physiques et de l'obstacle linguistique. La conscience des incompréhensions réciproques autour du bon usage des mécanismes de coordination utilisés demeure très inégale. Les écarts de pratiques managériales observés sont plus souvent interprétés par les non-Français comme le fruit d'une supposée incompétence managériale et, par les Français, comme un manque d'investissement dans la construction d'un groupe.

La littérature managériale pointe de manière récurrente les « problèmes humains » et en particulier les divergences de cultures nationales ou organisationnelles responsables de l'échec de certains rapprochements d'entreprise à l'échelle internationale. Cependant, elle se garde le plus souvent d'examiner les conditions concrètes de réalisation des coordinations transversales quotidiennes que cette dimension internationale implique. La question de savoir comment organiser en pratique la coordination des contributions des différentes parties prenantes, bien que celles-ci possèdent chacune leur style de travail et leur conception des manières propres à assurer des coopérations efficaces n'est guère abordée. Au final, les dysfonctionnements de ces structures restent largement méconnus au sein même de l'entreprise et, plus particulièrement, des niveaux décideurs dont l'attention se concentre sur bien d'autres enjeux. Leur impact n'en reste pas moins conséquent non seulement sur la qualité des décisions prises mais aussi sur le moral des salariés, et leur désir de travailler ensemble.

\section{Une intégration fragilisée}

Aux difficultés partagées avec n'importe quelle structure transversale nationale s'ajoutent les handicaps identifiés de la langue, de la distance et des différences culturelles dont les acteurs ont très inégalement conscience. 
L'obstacle de la langue ne renvoie pas seulement aux difficultés que peuvent éprouver certains acteurs à communiquer. L'unification volontariste du vocabulaire expose les différentes composantes de l'entreprise internationale à de sérieux malentendus tant peuvent être intraduisibles certaines appellations de fonction (business owner, business community), ou les étapes d'un processus de décision (concertation, problem solving, mitbestimmung). Par-delà les difficultés de communication liées à la diversité des langues, les cultures façonnent les représentations des difficultés rencontrées et orientent l'imputation des responsabilités attenantes. Le dysfonctionnement des structures de coordination affecte in fine l'implication des acteurs et la vision qu'ils ont du groupe. La coordination par la « culture de groupe », s'éloigne. En lieu et place de l'intégration progressive espérée, ce sont les stéréotypes culturels sur les Français qui prospèrent dans les filiales.

\section{IV - QUE FAIRE?}

Comment gérer l'inconfort d'une telle situation? La tentation existe au sein des entreprises multinationales de codifier dans un document unique les attitudes et les comportements qu'elle souhaiterait voir adopter par tous ses membres. Cette standardisation de la culture serait, à notre sens, aussi illusoire que contre-productive. Illusoire, en l'absence d'instances de contrôle capables de veiller sur l'application de ces normes; contre-productive dans la mesure où, loin d'apporter aux différents acteurs de base les éclairages escomptés sur les logiques d'action de leurs partenaires, elle les confronterait à une déstabilisation supplémentaire. Il $\mathrm{y}$ a, nous semble-t-il, mieux à faire.
Sensibiliser les acteurs aux incompréhensions que leurs propres conduites peuvent susciter paraît fort utile pour les aider à bâtir des arrangements mutuellement acceptables, nourris d'une compréhension croisée de l'originalité de chacun. Les Français, volontiers universalistes, peuvent s'appliquer utilement la démarche à euxmêmes. Tant qu'ils n'auront pas pris conscience de la singularité de leurs pratiques, tant qu'ils n'auront donc pas été en mesure d'aider leurs partenaires à décoder cette originalité dans les manières de décider, déléguer ou traiter un client, ils seront exposés à se voir opposer, de façon évidemment péjorative, la distance qui bien souvent sépare leurs manières de faire des « standards internationaux ».

Ce qui, dans notre recherche, s'applique aux Français est de portée plus générale. Les différentes parties prenantes de ces structures informelles de coordination ont besoin, pour travailler efficacement ensemble, de décoder la façon dont leurs nouveaux partenaires conçoivent leur travail, leur hiérarchie, leurs clients et leurs fournisseurs, leur entreprise et sa direction. Un travail réflexif sur les difficultés rencontrées dans le déroulement de leur collaboration les aiderait tout autant, sinon davantage, que les sensibilisations à l'interculturel auxquelles, dans le meilleur des cas, quelques-uns d'entre eux ont eu droit. Pareille démarche n'est évidemment pas sans coût, tant en moyens qu'en temps, cette dernière dimension n'étant, de l'avis des intéressés, pas la moindre. Aux firmes multinationales d'ajuster l'investissement qu'elles sont prêtes à engager en cette matière à la hauteur de leurs ambitions en matière d'intégration transnationale. 


\section{BibliograPhie}

Barmeyer C., Davoine E., « Chartes de valeurs et culture(s) de l'entreprise internationale: les limites du transfert de valeurs globales aux filiales », IAS: Audit social et responsabilité sociale et développement durable: vers une convergence européenne?, Luxembourg, 2004, p. 102-111.

Barsoux J.-L., Lawrence P., Management in France, Thomson Learning, 1990.

Clausen L., "Moving beyond stereotypes in managing cultural difference: Communication in Danish-Japanese corporate relationships", Scandinavian Journal of Management, $\mathrm{n}^{\circ} 26$, 2010, p. 57-66.

Clemmons S., Simon S.J., "Control and coordination in global ERP configuration", Business Process Management Journal, vol. 7, n 3, 2001, p. 205-215.

Huault I., Le management international, La Découverte \& Syros, Paris, 1998.

Iribarne P. (d'), Cultures et mondialisation, chapitre IV, Éditions du Seuil, Paris, 1998.

Iribarne P. (d'), L'épreuve des différences. L'expérience d'une entreprise mondiale, Éditions du Seuil, Paris, 2009.

Keup M., Gassmann O., "Why do transnational approaches to innovation fail?", Research in international Business and Finance, $\mathrm{n}^{\circ} 23,2009$, p. 206-222.

Kostova T., Roth K., "Adoption of an organizational practice by subsidiaries of multinational corporations: Institutional and relational effects", Academy of Management Journal, vol. 45, $\mathrm{n}^{\circ}$ 1, 2002, p. 215-233.

Lawrence P., Lorsch J., Adapter les structures de l'entreprise, Éd. d'Organisation, Paris, 1989 (traduction française).

Morgan G., "The Multinational Firm: Organizing Across Institutional and National Divides", The Multinational Firm, G. Morgan, P. H. Kristensen, R. Whitley, Oxford University Press, 2001.

Moennaert R.K., Caeldries F., Lievens A., Wauters E., "Communication flows in international product innovation teams", Journal of product innovation management, $\mathrm{n}^{\circ} 17,2000$, p. 360-377.

Moore F., Transnational Business Cultures, Ashgate, Hants, 2005.

Nohria N., Ghoshal S., The Differentiated Network: Organizing Multinational Corporations for Value Creation, Jossey-Bass, San Francisco, 1997.

Persson M., "The impact of operational structure, lateral integrative mechanisms on intraMNE knowledge transfer", International Business Review, vol. 15, 2006, p. 547-569.

Piekkari R., Vaara E., Tienari J., Säntti R., "Integration or disintegration? Human resource implications of a common corporate language decision in a crossborder merger", International Journal of Human Resource Management, vol. 16, $\mathrm{n}^{\circ}$ 3, 2005, p. 330-344.

Schaaper J. "Contrôle multidimensionnel d'une filiale à l'étranger: construction d'un modèle causal à partir du cas des multinationales européennes et japonaises en Chine », Finance, Contrôle, Stratégie, vol. 8, n 1, 2005, p. 159-190. 